\title{
Mechanisms of macrophage activation in obesity-induced insulin resistance
}

\author{
Justin I Odegaard ${ }^{1}$ and Ajay Chawla ${ }^{2}$ \\ ${ }^{1}$ Graduate Program in Immunology, Department of Medicine, Stanford University School of \\ Medicine, Stanford, California 94305-5103, USA \\ ${ }^{2}$ Division of Endocrinology, Metabolism and Gerontology, Department of Medicine, Stanford \\ University School of Medicine, Stanford, California 94305-5103, USA
}

\section{SUMMARY}

Chronic inflammation is now recognized as a key step in the pathogenesis of obesity-induced insulin resistance and type 2 diabetes mellitus. This low-grade inflammation is mediated by the inflammatory (classical) activation of recruited and resident macrophages that populate metabolic tissues, including adipose tissue and liver. These findings have led to the concept that infiltration and activation of adipose tissue macrophages is causally linked to obesity-induced insulin resistance. Studies have shown, however, that alternatively activated macrophages taking residence in adipose tissue and liver perform beneficial functions in obesity-induced metabolic disease. By attenuating tissue inflammation and increasing oxidative metabolism in liver and skeletal muscle, alternatively activated macrophages have lessened insulin resistance in obese mice. The discovery that distinct subsets of macrophages are involved in the promotion or attenuation of insulin resistance suggests that pathways controlling macrophage activation can potentially be targeted to treat these co-morbidities of obesity. Thus, this Review focuses on the stimuli and mechanisms that control classical and alternative activation of tissue macrophages, and how these macrophage activation programs modulate insulin action in peripheral tissues. The functional importance of macrophage activation is further discussed in the context of host defense to highlight the crosstalk between innate immunity and metabolism.

\section{Keywords}

alternatively activated macrophages; inflammation; insulin resistance; obesity; peroxisome proliferator-activated receptors

\section{INTRODUCTION}

Sedentary lifestyles and western diets are changing the face of chronic disease in developed and developing countries. Obesity associated with these lifestyle changes is increasing at an

Correspondence, Ajay Chawla, 300 Pasteur Dr., Endocrinology, S025, SUMC, Stanford, CA 94305-5103, USA; achawla@stanford.edu.

Justin Odegaard is a MD/PhD student at Stanford University School of Medicine; Ajay Chawla is an Assistant Professor of Medicine at Stanford University.

Competing Interests

The authors declared no competing interests.

\section{REVIEW CRITERIA}

Original articles on the role of inflammation and macrophages in obesity-induced insulin resistance published between 1982 and 2008 were identified in MEDLINE using the PubMed portal. The search terms used were "macrophages", "obesity", "insulin resistance" and "inflammation". All papers used in the review were in published in English language. 
alarming rate: currently, one in three adults in the US is obese, which is a $75 \%$ increase from $1980 ; 1^{1}$ the number of obese individuals worldwide has been estimated at 2.1 billion. ${ }^{2}$ As a result, the prevalence of obesity-related metabolic disorders, including insulin resistance, type 2 diabetes mellitus, dyslipidemia, atherosclerosis, hypertension, and fatty liver disease, is rising dramatically. In addition to accounting for 6-7\% of all-cause mortality in the US, ${ }^{3}$ the annual health-care costs for obesity-related disorders are estimated to be in excess of $\$ 70$ billion. ${ }^{4}$ Furthermore, if obesity and type 2 diabetes rates continue to increase unabated, especially in children, US life expectancy is predicted to plateau or decrease in the first half of this century. ${ }^{5}$ Thus, to blunt the eventual health and financial impact of this epidemic on our society, it is extremely important to understand how obesity leads to insulin resistance and type 2 diabetes. In this Review, we will provide a conceptual framework for understanding the dynamic crosstalk between the immune system and peripheral metabolism, and specifically discuss how obesity leads to the macrophage recruitment and activation that causes insulin resistance.

\section{OBESITY-INDUCED INSULIN RESISTANCE}

Studies with in humans and mice indicate that type 2 diabetes is a polygenic disease involving peripheral insulin resistance and impaired insulin secretion by pancreatic $\beta$ cells. ${ }^{6}$ Gene knockout studies in mice have shown that the insulin receptor, insulin receptor substrates, glucose transporter 4, peroxisome proliferator-activated receptors (PPARs), oxidative phosphorylation, and retinol binding protein have key roles in the development of insulin resistance can dramatically modify the phenotypic expression of disease. ${ }^{7-10}$ Environmental factors, such as dietary intake and energy expenditure also have notable effects. These findings suggest that increase in adiposity somehow physiologically interacts with the genetic parameters that control insulin sensitivity. Indeed, chronic, low-grade inflammation provides a molecular pathway that links obesity to insulin resistance, and its sequela, coronary artery disease. ${ }^{11,12}$ Interestingly, dysregulation of innate immunity, specifically activation of tissue macrophages, is primarily responsible for the observed increase in tissue inflammation in obese mice and humans. ${ }^{13,14}$

\section{CROSSTALK BETWEEN METABOLISM AND IMMUNITY}

The role of inflammation in insulin resistance has become well established during the past decade of intensive research but the complex crosstalk between the immune system and metabolic tissues has been recognized only in the past five years. To fully appreciate the molecular dialogue between these essential systems, it is necessary to first review the mechanisms by which the immune system protects the host against attacks. In vertebrates, a layered immune defense confers protection against a myriad of invading pathogens, ranging from viruses to parasites. Cells of the innate immune system, including macrophages, neutrophils, and dendritic cells, provide the first-line defense against pathogens that breach surface barriers. ${ }^{15}$ Recognition of pathogens via germline-encoded pattern-recognition receptors initiates signaling that leads to activation of NF- $\mathrm{KB}$, a transcription factor required for expression of various proinflammatory and antimicrobial programs.${ }^{15,16}$ In contrast to the immediate response of innate immune cells, activation of adaptive immune responses occurs 4-7 days later, and is characterized by clonal expansion of T cells and $\mathrm{B}$ cells. These $\mathrm{T}$ cells and $\mathrm{B}$ cells express somatically rearranged receptors, thus conferring antigen-specific and pathogen-specific, long-lasting immunity. ${ }^{17}$ Although studies have thus far focused on the role of the innate immune system in obesity-induced insulin resistance, it seems likely that molecules elaborated by the adaptive immune system might also regulate nutrient homeostasis and insulin action. 
The anatomic juxtaposition of immune and metabolic cell types provides an architectural framework for direct communication between these two fundamental systems. The majority of metabolic organs are densely populated with cells of the innate and adaptive immune system. For instance, liver and white adipose tissues both contain an abundant number of macrophages, whose activation status changes with obesity. ${ }^{18-21}$ In addition, various other immune cell types, including T and B lymphocytes, natural killer T cells, neutrophils, and dendritic cells, are resident in these tissues, and their numbers are known to change during obesity. ${ }^{22}$ For instance, while obesity induces a dramatic decrease in the number of natural killer $\mathrm{T}$ cells in the liver, ${ }^{23}$ the number of activated $\mathrm{T}$ cells increases in the white adipose tissue. $^{24}$

Adipose tissue and liver also provide the nutrients necessary to sustain activation of innate and adaptive immune responses. ${ }^{25}$ Congruent with this idea, nearly all lymph nodes are embedded in depots of white adipose tissue, which provide a dedicated lipid source for fueling immune activation. ${ }^{26}$ In addition, liver and white adipose tissues are the likely sources for the nutrients, glucose, and fatty acids, that sustain the proliferative and secretory programs of activated lymphocytes and myeloid cells, respectively. Indeed, immune activation rapidly and substantially augments this systemic metabolic output. ${ }^{27}$ Thus, rapid changes in nutrient flux seem not only to accompany but also to be necessary for immune activation, implying that signals elaborated by immune cells might directly regulate systemic metabolism. Indeed, studies have highlighted a key role for activated macrophages in controlling energy metabolism and insulin action. These topics will be discussed in further detail below.

\section{RECRUITMENT AND ACTIVATION OF ADIPOSE TISSUE MACROPHAGES}

Macrophages are a highly heterogeneous cell type found in nearly all tissues of the body. ${ }^{28}$ Generally, these cells have been deemed immune sentinels that quiescently monitor the tissue milieu for early signs of infection or damage. In this role, the macrophage is responsible for sensing, integrating, and responding appropriately to a bewildering array of stimuli from its microenvironment. These pleiotropic responses are coordinated through distinct programs of macrophage activation, classified as classical (or M1) to alternative (or M2) OK. ${ }^{29-31}$ Stimulation of macrophages with T-helper-1-type cytokines, such as interferon $\gamma$, or bacterial by-products promotes maturation of classically activated macrophages with high inflammatory and bactericidal potential. By contrast, T-helper-2type cytokines, including interleukins 4 and 13, promote alternative activation of macrophages, which have known antiparasitic functions and ill-defined functions in tissue repair and remodeling (Figures 1 and 2). Although a variety of markers are differentially expressed in these macrophage subsets (Figure 1) ${ }^{30}$ the differential metabolism of Larginine provides the most consistent means of distinguishing these two activation states. ${ }^{32,33}$ While classically activated macrophages upregulate inducible nitric oxide synthase to catabolize L-arginine to nitric oxide and citrulline, alternatively activated macrophages preferentially induce arginase I, which metabolizes arginine to ornithine and polyamines, precursors necessary for collagen synthesis and cellular proliferation, respectively. ${ }^{30}$

As mentioned above, a chronic, low-grade inflammatory state contributes to the comorbid conditions associated with obesity, such as insulin resistance, type 2 diabetes, dyslipidemia, and atherosclerosis. For instance, in obese animals, adipose tissue secretes various proinflammatory molecules, including tumor necrosis factor (TNF) $\mathbf{O K}$, interleukin 6, and reactive nitrogen species, which directly or indirectly interfere with the insulin signaling pathway. ${ }^{34}$ Congruent with these findings, genetic deletion of TNF or inducible nitric oxide synthase greatly attenuates insulin resistance in obese mice. ${ }^{35,36}$ 
Although the enlarging adipocytes were originally thought to be the source of proinflammatory molecules, studies in mice have suggested that bone-marrow-derived macrophages are the major culprits. ${ }^{18}$ Consistent with this idea, impaired recruitment of macrophages to adipose tissue, as occurs in the $\mathrm{C}-\mathrm{C}$ motif chemokines receptor 2 or osteopontin knockout mice, reduces inflammation in adipose tissue and improves insulin sensitivity in obese mice. ${ }^{37}$ By contrast, adipocyte-specific transgenic expression of monocyte chemoattractant protein 1 , the chemokine ligand for $\mathrm{C}-\mathrm{C}$ motif chemokines receptor 2 , increases recruitment of proinflammatory, classically activated macrophages and potentiates insulin resistance. ${ }^{38,39}$ These findings suggest a paradigm in which infiltration of adipose tissue with macrophages is linked to the development of obesity-induced insulin resistance.

\section{CLASSICALLY ACTIVATED MACROPHAGES PROMOTE INSULIN RESISTANCE}

Analysis of macrophages that infiltrate white adipose tissue of obese mice suggests that these cells predominantly express the classically activated phenotype. ${ }^{13,14}$ These observations raise the key question of what stimuli promote the proinflammatory activation of adipose tissue macrophages. Potential insights can be derived from closer inspection of the innate immune response to pathogens. Ligation of Toll-like receptor 4 (TLR4) by bacterially derived lipids, such as lipopolysaccharides, is known to activate inflammatory signaling pathways that induce NF- $\kappa \mathrm{B}$ to trigger release of various inflammatory molecules, including TNF, interleukins 6 and 12, and inducible nitric oxide synthase (Figure 2). ${ }^{16}$ The same innate immune module becomes activated in obesity-induced inflammation and insulin resistance (Figure 2). Instead of lipopolysaccharide, in obesity saturated fatty acids, such as myristic, palmitic and stearic No fatty acids, activate TLR4 to drive the inflammatory activation of tissue macrophages. ${ }^{40-42}$ Consistent with this notion, acute infusion of lipids into mice potentiates insulin resistance in both adipose tissue and skeletal muscle in a TLR4dependent manner. ${ }^{40}$ Absence of TLR4 confers only partial protection to female mice from inflammation and insulin resistance induced by eating a high-fat diet, suggesting potential involvement of other sex-specific factors, whose identity remains unknown.

In addition to TLR4 signaling, chronic overnutrition activates other protein kinases, including I $\mathrm{kB}$ kinase $\beta$ ([IKK $\beta]$ encoded by Ikbkb in mice) and Jun kinase 1 (JNK1, encoded by Mapk8), which promote insulin resistance by distinct mechanisms. ${ }^{34}$ Activation of the stress kinase JNK1 results in inhibitory serine phosphorylation of insulin receptor substrate 1 , leading to decreased tyrosine kinase signaling from the insulin receptor. By contrast, IKK $\beta$ activation during obesity releases NF- $\kappa$ B from its inhibitor $\mathrm{I} \kappa \mathrm{B}$, resulting in increased tissue inflammation. Consistent with their respective roles in stress and inflammatory signaling, global or hepatic deletion of Mapk 8 and $I k b k b$, respectively, has a profound effect on obesity-induced metabolic disease. While Mapk 8 knockout mice are protected from diet-induced obesity and, indirectly, insulin resistance, ${ }^{43}$ hepatic deletion of $I k b k b$ prevents the development of inflammation and insulin resistance in this depot. ${ }^{44,45}$ These data demonstrate that activation of JNK1 and IKK $\beta$ in metabolic tissues is, in part, responsible for the onset of insulin resistance in obese animals.

In addition to their role in metabolic tissues, IKK $\beta$ and JNK1 seem to be required for classical activation of resident macrophages during obesity. ${ }^{44}$ The contribution of myeloid cell-specific IKK $\beta$ and hematopoietic cell-specific JNK1 to obesity and insulin resistance have been investigated by two different approaches. For the studies on IKK $\beta$ mice with a cre gene knocked into the lysozyme 2 gene (LysM-cre) ok were used to delete the floxed IKK $\beta$ genes in all myeloid cells, including macrophages and neutrophils. ${ }^{44}$ As expected, mice deficient in IKK $\beta$ in myeloid cells had lower levels of tissue inflammation and preserved 
insulin sensitivity when receiving the high-fat diet. For analysis of JNK1 activity in hematopoietic and nonhematopoietic compartments, Solinas ok and colleagues ${ }^{46}$ adoptively transferred bone marrow between wild type and Mapk 8 knockout mice. Analyses of these chimeric animals revealed that absence of $M a p k 8$ in nonhematopoietic cells protects mice from obesity induced by a high-fat diet. By contrast, removal of Mapk 8 from the hematopoietic compartment decreased hepatic and adipose tissue inflammation, leading to improvement in insulin resistance. Interestingly, stimulation of peritoneal macrophages or Kupffer cells with high concentrations of saturated fatty acids led to sustained activation of JNK1, which was necessary for classical activation of tissue macrophages. Together, these data suggest a model in which increased flux of saturated fatty acids, as seen in obese states, activates NF- $\mathrm{kB}$ and JNK1 kinases in resident macrophages, leading to classical macrophage activation, tissue inflammation, and insulin resistance (Figure 2).

\section{ALTERNATIVELY ACTIVATED MACROPHAGES PROTECT AGAINST INSULIN RESISTANCE}

Although the number of classically activated adipose tissue macrophages increases with obesity, adipose tissue of lean animals also contains a moderate number of macrophages. Adipose tissue macrophages from lean mice express high levels of arginase 1, mannose receptor, dectin-1, Ym1, and macrophage galactose $N$-acetyl-galactosamine-specific lectins 1 and 2, all of which are encoded by signature genes of alternatively activated macrophages (Figure 1). ${ }^{20,21}$ Since obesity leads to recruitment of classically activated macrophages, it results in a net shift in the activation state of adipose tissue macrophages from alternative to classical. Furthermore, based on the anti-inflammatory activities of alternatively activated macrophages, it has been suggested that these cells might confer protection from the metabolic sequelae of obesity; however, definitive proof for this action is lacking.

To explore the therapeutic potential of alternatively activated macrophages in metabolic disease, we have undertaken a systematic approach to identify the transcriptional switches that control alternative macrophage activation. These studies have revealed that regulators of fatty acid metabolism play key parts in the acquisition and maintenance of the alternative state. ${ }^{47}$ While the T-helper- 2 cytokine interleukin 4 and the signal transducer and activator of transcription 6 (STAT6) provide the initial instructions for alternative macrophage activation, PPARs, the body's sensors of fatty acids, ${ }^{9}$ are needed to sustain its expression (Figure 3). In support of this idea, PPAR $\gamma$ and PPAR $\delta$ have been shown to bind directly to and activate the macrophage-specific enhancer of arginase 1, a hallmark of alternatively activated macrophages. ${ }^{21,48,49}$ While PPAR $\gamma$ is required for the metabolic programming of alternatively activated macrophages, ${ }^{21}$ PPAR $\delta$ seems to be important for coordinating the immune programs of these cells, including expression of pattern recognition receptors (encoded by $\mathrm{Mrc1}$ and $\mathrm{Clec} 7 \mathrm{a}$ ) and co-stimulatory molecules (Pdcd1Ig2), and suppression of macrophage-inflammatory response. ${ }^{48}$ Importantly, we discovered that monounsaturated fatty acids, such as oleic acid, synergize with interleukin 4 in a PPAR $\delta$-dependent manner to drive the macrophage program of alternative activation. Taken together with the previous findings on saturated fatty acids and classical macrophage activation, these data suggest a model in which the local milieu of fatty acids dictates the activation state of tissue macrophages; saturated fatty acids, such as palmitic acid, trigger TLR4/NF- $\kappa B$ and JNK1 signaling pathways to promote classical activation of macrophages. Monounsaturated fatty acids, such as oleic acid, however, ligate PPARs to polarize resident macrophages to the anti-inflammatory alternative state (Figure 2).

By use of mouse models in which alternative activation of tissue macrophages was severely compromised, Odegaard et al..$^{21,48}$ investigated the functions of these cells in obesityinduced metabolic disease. For studies involving $\operatorname{PPAR} \gamma$, macrophage-specific PPAR $\gamma$ 
knockout mice were generated on the T-helper-2-permissive Balb/c strain by interbreeding PPAR $\gamma$ floxed and LsyM-Cre mice. ${ }^{21}$ Expression analysis from lean and obese mice demonstrated that macrophage-specific PPAR $\gamma$ knockout mice had dramatically lower numbers of alternatively activated adipose tissue macrophages. Strikingly, feeding these mice a high-fat diet resulted in moderate obesity and insulin resistance in liver and skeletal muscle, ${ }^{21,50}$ suggesting that alternatively activated adipose tissue macrophages confer protection against the development of metabolic syndrome.

To assess the contribution of PPAR $\delta$ in alternative macrophage activation and diet-induced metabolic disease, bone marrow from wild type or PPAR $\delta$-null mice was transplanted into wild type animals. ${ }^{48}$ Analyses of these chimeric animals revealed that hematopoietic deficiency of PPAR $\delta$ increased both adiposity and peripheral insulin resistance. In marked contrast to what was observed in macrophage-specific PPAR $\gamma$ knockout mice, adoptive transfer of PPAR $\delta$-null bone marrow primarily affected alternative activation of Kupffer cells, the resident population of macrophages present in the liver, while sparing the adipose tissue macrophages. ${ }^{48}$ Similarly, Kang et al. ${ }^{51}$ observed that deletion of PPAR $\delta$ in myeloid cells decreased alternative activation potential of adipose tissue macrophages, resulting in more severe obesity-induced insulin resistance. In aggregate, these findings suggest that, in a depot specific manner, PPARS and PPAR $\gamma$ regulate alternative activation of adipose tissue macrophages and Kupffer cells, respectively, to ameliorate obesity-induced insulin resistance.

A key question that emerges from this work is how alternatively activated macrophages regulate parenchymal cell metabolism. One potential explanation would be that factors released by alternatively activated macrophages act in a paracrine manner to modulate insulin action or cellular metabolism of the parenchymal cells. In support of this, coculture of adipocytes with PPAR $\gamma$-deficient macrophages led to a dramatic reduction in insulinstimulated glucose uptake, suggesting that alternatively activated adipose tissue macrophages play an essential part in maintaining adipocyte function. ${ }^{21,50}$ Moreover, deficiency of alternatively activated macrophages in adipose tissue or liver was associated with downregulation of cellular programs for oxidative phosphorylation and $\beta$-oxidation in skeletal muscle and liver. Remarkably, this suppressive effect on $\beta$-oxidation could be recapitulated by treating primary hepatocytes with conditioned medium from PPAR $\delta$-null macrophages, implying that factors elaborated by alternatively activated macrophages improve the ability of tissues to use incoming fatty acids. ${ }^{48,51}$ While these findings substantially advance our understanding of the dynamic crosstalk that exists between resident alternatively activated macrophages and metabolic tissues, additional investigations will be necessary to identify the molecules secreted by macrophages that relay this information to the metabolic tissues in a paracrine or endocrine fashion.

\section{CONCLUSIONS}

The collective work on classically and alternatively activated macrophages suggests that macrophage activation plays a dynamic role in modulating insulin sensitivity in animals. Since it is unlikely that good (alternatively activated) and bad (classically activated) macrophages were selected for metabolic disease, one must ask why is it that these two macrophage activation states have such a profound effect on insulin action and, indirectly, glucose homeostasis? A potential explanation might be that the decrease in insulin sensitivity that occurs with classical macrophage activation is necessary for proper execution of host defense functions. For instance, during acute bacterial infection, such as with gram negative rods, activation of the TLR4/NF- $\kappa \mathrm{B}$ module increases respiratory and inflammatory burst of macrophages, programs necessary for the rapid killing of invading pathogens (Figure 2). While absolutely necessary for host survival, this inflammatory 
program of macrophage activation is bioenergetically demanding and primarily fueled by glycolysis. ${ }^{52,53}$ Because macrophages and neutrophils lack substantial stores of glucose and glycogen, they rely heavily on circulating nutrients to meet their metabolic needs. In support of this viewpoint, TNF increases release of free fatty acids from adipocytes, ${ }^{54}$ whereas IL-6 and IL-1a augment fatty acid oxidation and oxidative metabolism in skeletal muscle. ${ }^{55,56}$ Thus, in this context, inflammation-induced insulin resistance is an adaptive response that allows the immune system to have unfettered access to nutrients, including glucose and amino acids, at the expense of peripheral tissues. In the setting of obesity, however, chronic activation of this adaptive response becomes maladaptive, predisposing mice to obesityinduced insulin resistance and metabolic syndrome (Figure 2).

A similar paradigm can be invoked for the regulation of peripheral metabolism by alternatively activated macrophages. Rather than conferring protection against bacteria, the T-helper-2 component of the immune system primarily provides a defense against parasites (Figure 2). Since this T-helper-2 immune response is often elicited during the chronic phase of helminth infection, it has been postulated that in this setting, the metabolic demands of the immune system are rather different, both in terms of intensity and duration. Accordingly, expression and metabolic profiling of activated macrophages has identified that T-helper-2 cytokines induce oxidative metabolism, thereby shifting reliance from glycolysis to fatty acid oxidation for ATP production. ${ }^{21,47}$ Moreover, since factors elaborated by alternatively activated macrophages increase oxidative metabolism in peripheral tissues, ${ }^{21,48,51}$ induction of T-helper-2 immunity will prevent build up of cytosolic fatty acids and its metabolites, such as acetyl-coenzyme As and diacylglycerol. This shift to oxidative metabolism will have the net effect of reducing lipotoxic effects in liver and skeletal muscle, thereby leading to improvement in insulin action. ${ }^{57}$ The bioenergetic demands of an activated immune system drive the changes in insulin sensitivity, which, in this case, result in improvement of glucose homeostasis in obese mice. Thus, this biological interface between innate immunity and metabolism provides new cellular and molecular targets that can be therapeutically exploited for treatment of co-morbidities of obesity, such as insulin resistance, T2DM and atherosclerosis.

\section{Acknowledgments}

We thank A Loh for critical reading of the manuscript. This work was supported by grants made available to AC: NIH (DK062386 and HL076746), Takeda Pharmaceuticals of North America and the American Diabetes Association A Chawla. Support was provided to JI Odegaard by Stanford Medical Scientist Training Program and the AHA.

\section{References}

1. Flegal KM, et al. Prevalence and trends in obesity among US adults 1999-2000. JAMA. 2002; 288:1723-1727. [PubMed: 12365955]

2. Li Z, et al. Health ramifications of the obesity epidemic. Surg Clin North Am. 2005; 85:681-701. v. [PubMed: 16061080]

3. Ford ES. Risks for all-cause mortality, cardiovascular disease, and diabetes associated with the metabolic syndrome: a summary of the evidence. Diabetes Care. 2005; 28:1769-1778. [PubMed: 15983333]

4. Allison DB, et al. The direct health care costs of obesity in the United States. Am J Public Health. 1999; 89:1194-1199. [PubMed: 10432905]

5. Olshansky SJ, et al. A potential decline in life expectancy in the United States in the 21st century. N Engl J Med. 2005; 352:1138-1145. [PubMed: 15784668]

6. Stumvoll M, et al. Type 2 diabetes: principles of pathogenesis and therapy. Lancet. 2005; 365:13331346. [PubMed: 15823385] 
7. Kadowaki T. Insights into insulin resistance and type 2 diabetes from knockout mouse models. J Clin Invest. 2000; 106:459-465. [PubMed: 10953020]

8. Yang Q, et al. Serum retinol binding protein 4 contributes to insulin resistance in obesity and type 2 diabetes. Nature. 2005; 436:356-362. [PubMed: 16034410]

9. Evans RM, et al. PPARs and the complex journey to obesity. Nat Med. 2004; 10:355-361. [PubMed: 15057233]

10. Lin J, et al. Metabolic control through the PGC-1 family of transcription coactivators. Cell Metabolism. 2005; 1:361-370. [PubMed: 16054085]

11. Shoelson SE, et al. Inflammation and insulin resistance. J Clin Invest. 2006; 116:1793-1801. [PubMed: 16823477]

12. Hotamisligil GS. Inflammation and metabolic disorders. Nature. 2006; 444:860-867. [PubMed: 17167474]

13. Bouloumié A, et al. Role of macrophage tissue infiltration in metabolic diseases. Curr Opin Clin Nutr Metab Care. 2005; 8:347-354. [PubMed: 15930956]

14. Ferrante AW Jr. Obesity-induced inflammation: a metabolic dialogue in the language of inflammation. J Intern Med. 2007; 262:408-414. [PubMed: 17875176]

15. Medzhitov R, Janeway C Jr. Innate immunity. N Engl J Med. 2000; 343:338-344. [PubMed: 10922424]

16. Takeda K, et al. Toll-like receptors. Annu Rev Immunol. 2003; 21:335-376. [PubMed: 12524386]

17. Medzhitov R, Janeway CA Jr. Innate immune recognition and control of adaptive immune responses. Semin Immunol. 1998; 10:351-353. [PubMed: 9799709]

18. Weisberg SP, et al. Obesity is associated with macrophage accumulation in adipose tissue. J Clin Invest. 2003; 112:1796-1808. [PubMed: 14679176]

19. $\mathrm{Xu} \mathrm{H}$, et al. Chronic inflammation in fat plays a crucial role in the development of obesity-related insulin resistance. J Clin Invest. 2003; 112:1821-1830. [PubMed: 14679177]

20. Lumeng CN, et al. Obesity induces a phenotypic switch in adipose tissue macrophage polarization. J Clin Invest. 2007; 117:175-184. [PubMed: 17200717]

21. Odegaard JI, et al. Macrophage-specific PPAR $\gamma$ controls alternative activation and improves insulin resistance. Nature. 2007; 447:1116-1120. [PubMed: 17515919]

22. Racanelli V, Rehermann B. The liver as an immunological organ. Hepatology. 2006; 43(Suppl 1):S54-S62. [PubMed: 16447271]

23. Li Z, et al. Dietary factors alter hepatic innate immune system in mice with nonalcoholic fatty liver disease. Hepatology. 2005; 42:880-885. [PubMed: 16175608]

24. Kintscher U, et al. T-lymphocyte infiltration in visceral adipose tissue: a primary event in adipose tissue inflammation and the development of obesity-mediated insulin resistance. Arterioscler Thromb Vasc Biol. 2008; 28:1304-1310. [PubMed: 18420999]

25. Fox CJ, et al. Fuel feeds function: energy metabolism and the T-cell response. Nat Rev Immunol. 2005; 5:844-852. [PubMed: 16239903]

26. Knight SC. Specialized perinodal fat fuels and fashions immunity. Immunity. 2008; 28:135-138. [PubMed: 18275823]

27. Wolowczuk I, et al. Feeding our immune system: impact on metabolism. Clin Dev Immunol. 2008; 2008:639803. [PubMed: 18350123]

28. Gordon S, Taylor PR. Monocyte and macrophage heterogeneity. Nat Rev Immunol. 2005; 5:953964. [PubMed: 16322748]

29. Goerdt S, et al. Alternative versus classical activation of macrophages. Pathobiology. 1999; 67:222-226. [PubMed: 10725788]

30. Gordon S. Alternative activation of macrophages. Nat Rev Immunol. 2003; 3:23-35. [PubMed: 12511873]

31. Martinez FO, et al. Macrophage activation and polarization. Front Biosci. 2008; 13:453-461. [PubMed: 17981560]

32. Modolell M, et al. Reciprocal regulation of the nitric oxide synthase/arginase balance in mouse bone marrow-derived macrophages by TH1 and TH2 cytokines. Eur J Immunol. 1995; 25:11011104. [PubMed: 7537672] 
33. Munder M, et al. Th1/Th2-regulated expression of arginase isoforms in murine macrophages and dendritic cells. J Immunol. 1999; 163:3771-3777. [PubMed: 10490974]

34. Hotamisligil GS. Inflammatory pathways and insulin action. Int J Obes Relat Metab Disord. 2003; 27(Suppl 3):S53-S55. [PubMed: 14704746]

35. Perreault M, Marette A. Targeted disruption of inducible nitric oxide synthase protects against obesity-linked insulin resistance in muscle. Nat Med. 2001; 7:1138-1143. [PubMed: 11590438]

36. Uysal KT, et al. Protection from obesity-induced insulin resistance in mice lacking TNF- a function. Nature. 1997; 389:610-614. [PubMed: 9335502]

37. Weisberg SP, et al. CCR2 modulates inflammatory and metabolic effects of high-fat feeding. J Clin Invest. 2005; 116:115-124. [PubMed: 16341265]

38. Kanda H, et al. MCP-1 contributes to macrophage infiltration into adipose tissue, insulin resistance, and hepatic steatosis in obesity. J Clin Invest. 2006; 116:1494-1505. [PubMed: 16691291]

39. Kamei N, et al. Overexpression of monocyte chemoattractant protein-1 in adipose tissues causes macrophage recruitment and insulin resistance. J Biol Chem. 2006; 281:26602-26614. [PubMed: 16809344]

40. Shi H, et al. TLR4 links innate immunity and fatty acid-induced insulin resistance. J Clin Invest. 2006; 116:3015-3025. [PubMed: 17053832]

41. Kim F, et al. Toll-like receptor-4 mediates vascular inflammation and insulin resistance in dietinduced obesity. Circ Res. 2007; 100:1589-1596. [PubMed: 17478729]

42. Nguyen MT, et al. A subpopulation of macrophages infiltrates hypertrophic adipose tissue and is activated by free fatty acids via Toll-like receptors 2 and 4 and JNK-dependent pathways. J Biol Chem. 2007; 282:35279-35292. [PubMed: 17916553]

43. Hirosumi J, et al. A central role for JNK in obesity and insulin resistance. Nature. 2002; 420:333336. [PubMed: 12447443]

44. Arkan MC, et al. IKK- $\beta$ links inflammation to obesity-induced insulin resistance. Nat Med. 2005; 11:191-198. [PubMed: 15685170]

45. Cai D, et al. Local and systemic insulin resistance resulting from hepatic activation of IKK- $\beta$ and NF-кB. Nat Med. 2005; 11:183-190. [PubMed: 15685173]

46. Solinas G, et al. JNK1 in hematopoietically derived cells contributes to dietinduced inflammation and insulin resistance without affecting obesity. Cell Metab. 2007; 6:386-397. [PubMed: 17983584]

47. Vats D, et al. Oxidative metabolism and PGC-1 $\beta$ attenuate macrophage-mediated inflammation. Cell Metab. 2006; 4:13-24. [PubMed: 16814729]

48. Odegaard JI, et al. Alterantive M2 activation of Kupffer cells by PPAR $\delta$ ameliroates insulin resistance. Cell Metab. 2008; 7:496-507. [PubMed: 18522831]

49. Gallardo-Soler A, et al. Arginase I induction by modified lipoproteins in macrophages: a PPAR $\gamma /$ $\delta$-mediated effect that links lipid metabolism and immunity. Mol Endocrinol. 2008; 22:13941402. [PubMed: 18323470]

50. Hevener AL, et al. Macrophage PPAR $\gamma$ is required for normal skeletal muscle and hepatic insulin sensitivity and full antidiabetic effects of thiazolidinediones. J Clin Invest. 2007; 117:1658-1669. [PubMed: 17525798]

51. Kang K, et al. Adipocyte-derived Th2 cytokines and myeloid PPARd regulate macrophage polarization and insulin sensitivity. Cell Metab. 2008; 7:485-495. [PubMed: 18522830]

52. Newsholme P, Newsholme EA. Rates of utilization of glucose, glutamine and oleate and formation of end-products by mouse peritoneal macrophages in culture. Biochem J. 1989; 261:211-218. [PubMed: 2775207]

53. Cramer T, et al. HIF-1a is essential for myeloid cell-mediated inflammation. Cell. 2003; 112:645657. [PubMed: 12628185]

54. Cawthorn WP, Sethi JK. TNF-alpha and adipocyte biology. FEBS Lett. 2008; 582:117-131. [PubMed: 18037376]

55. Puigserver $\mathrm{P}$, et al. Cytokine stimulation of energy expenditure through p38 MAP kinase activation of PPARgamma coactivator-1. Mol Cell. 2001; 8:971-982. [PubMed: 11741533] 
56. Bruce CR, Dyck DJ. Cytokine regulation of skeletal muscle fatty acid metabolism: effect of interleukin-6 and tumor necrosis factor-alpha. Am J Physiol Endocrinol Metab. 2004; 287:E616E621. [PubMed: 15149950]

57. Lowell BB, Shulman GI. Mitochondrial dysfunction and type 2 diabetes. Science. 2005; 307:384387. [PubMed: 15662004] 


\section{KEY POINTS}

Obesity, macrophage-mediated inflammation and insulin resistance

Dual functions of macrophages in obesity-induced insulin resistance

Obesity induces a switch in macrophage activation

Classically activated macrophages potentiate insulin resistance.

Alternatively activated macrophages confer protection from obesity and insulin resistance.

PPAR $\gamma$ and PPAR $\delta$ regulate alternative activation of macrophages. 


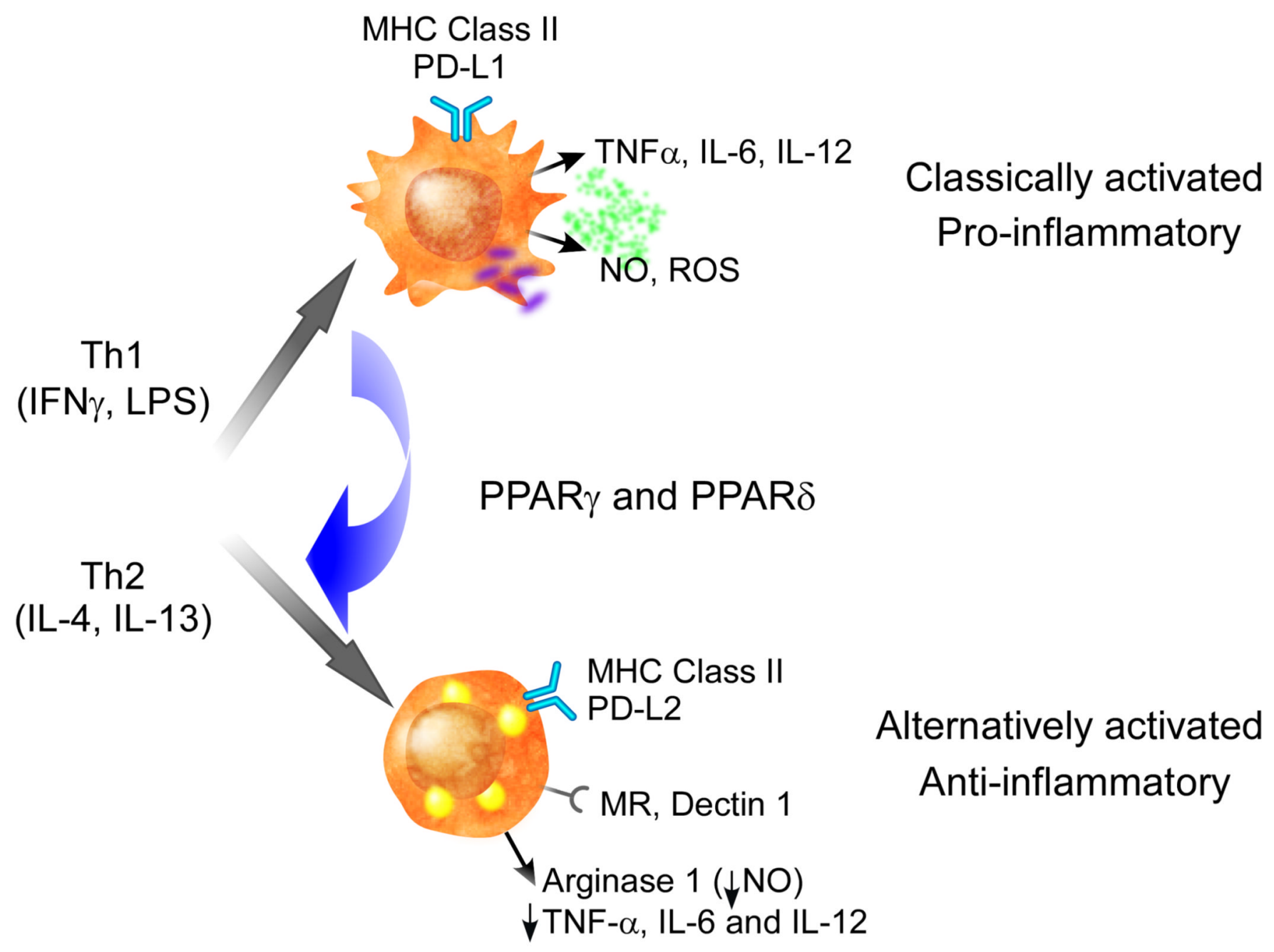

Figure 1.

Classical and alternative activation of macrophages. Microbial stimuli, which are recognized by pattern recognition receptors, and the T-helper-1 cytokine interferon $\gamma$ promote classical activation of resident and recruited macrophages. These activated cells produce proinflammatory cytokines, such as tumor necrosis factor and interleukin 6 , and reactive oxygen species and nitric oxide. Induction of major histocompatability complex class II and co-stimulatory molecules increases antigen presentation by these cells. Classically activated macrophages play an essential part in antibacterial responses. By contrast, interleukins 4 and 13 promote alternative macrophage activation, which plays a critical role in allergic and antiparasitic responses. Although these cells also induce major histocompatability complex class II, their repertoire of co-stimulatory molecules and pattern recognition receptors is different. The co-stimulatory molecule program death ligand 2 and pattern recognition receptors, mannose receptor and dectin-1, are highly induced in alternatively activated macrophages. Induction of arginase 1 diverts arginine catabolism away from nitric oxide synthesis to ornithine and urea. In addition, in a paracrine and autocrine manner, alternatively activated macrophages suppress inflammation. Abbreviations: IFN, interferon; IL, interleukin; LPS, lipopolysaccharide; MHC, major histocompatability complex; MR, mannose receptor; NO, nitric oxide; PD-L, program death ligand; PPAR, peroxisome proliferator-activated receptor; ROS, reactive oxygen species; $\mathrm{T}_{\mathrm{H}}$, T helper; TNF, tumor necrosis factor. 


\section{Stimuli}

A)

\author{
Bacteria
}

Lipids

(saturated FAs)

B) Parasites

Lipids
(unsaturated FAs)
Sensor

Macrophages

(TLR4-Myd88-NF-кB)

\author{
Macrophages \\ (TLR4/JNK/NF-кB)
}

\section{Effector}

Respiratory/

Inflammatory burst

Insulin resistance

\section{Alternatively activated (IL-4R/STAT6/PPAR $\gamma /$ PPAR $\delta$ )}

Attenuate Inflammation
Insulin sensitivity

Figure 2.

Innate immune modules deployed in host defense and obesity. Sensing of bacteria by the Toll-like receptor 4 leads to activation of NF- $\mathrm{BB}$, resulting in inflammatory activation of macrophages. In obesity, ligation of Toll-like receptor 4 and activation of JNK1 by saturated fatty acids, such as myristic, palmitic andstearic No acids, causes classical activation of tissue macrophages, leading to inflammation and insulin resistance. Parasitic infections result in activation of T-helper-2 cytokine signaling pathways in macrophages to promote alternative macrophage activation, which counteracts T-helper-1-type inflammation and facilitates containment of parasites. In contrast to saturated fatty acids, monounsaturated fatty acids, such as oleic acid, activate peroxisome proliferator-activated receptors $\delta$ and $\gamma$, and synergize with T-helper- 2 cytokines to polarize tissue macrophages to the alternative state. Factors released by alternatively activated macrophages attenuate tissue inflammation and promote parenchymal cell oxidative metabolism, resulting in insulin sensitization of metabolic tissues. Abbreviations: IL, interleukin; JNK, jun kinase; Myd88, Myeloid differentiation primary response gene; PPAR, peroxisome proliferator-activated receptor; STAT, signal transducer and activator of transcription; TLR4, Toll-like receptor 4. 


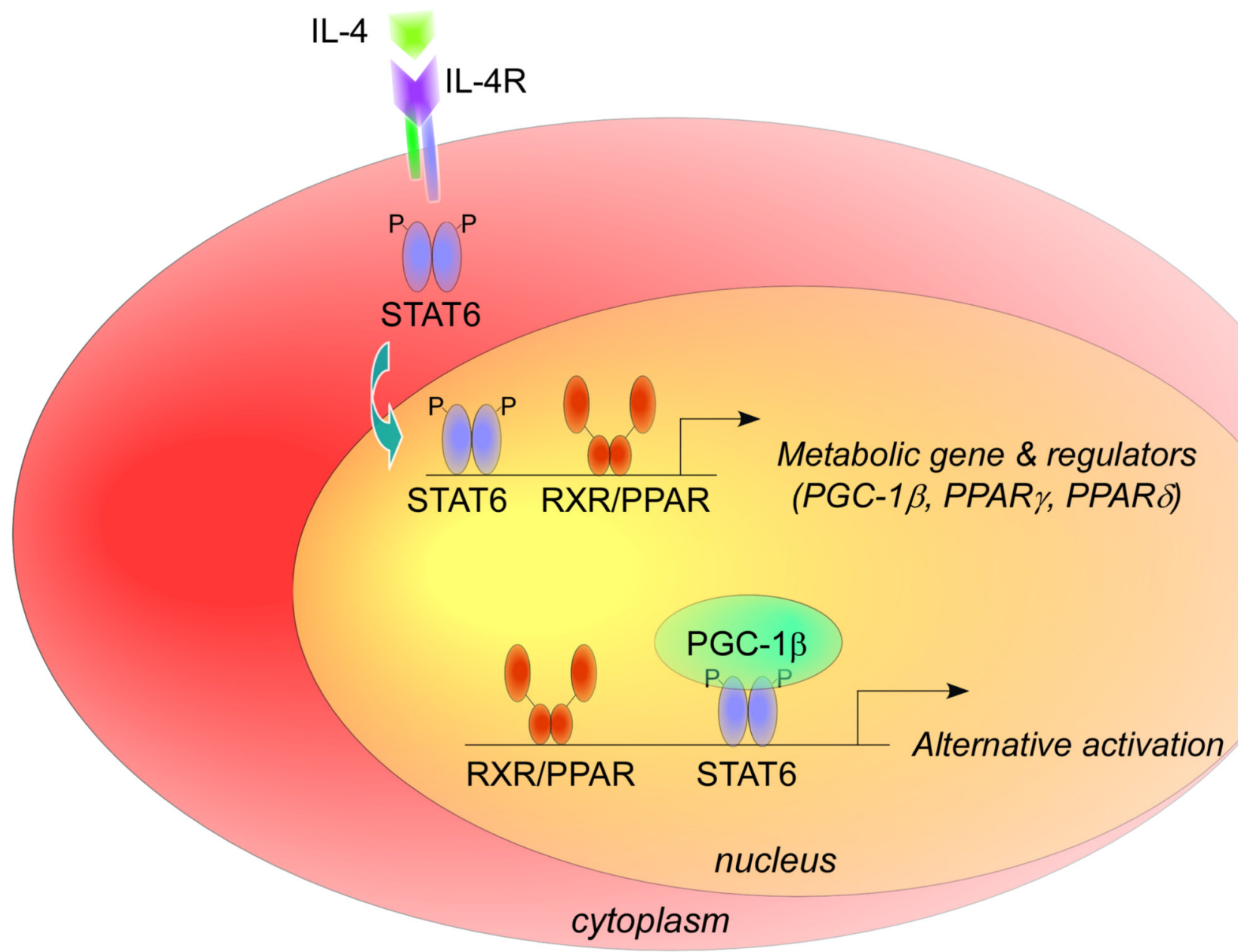

Figure 3.

Transcriptional model for alternative macrophage activation. Stimulation of macrophages with the T-helper- 2 cytokine interleukin 4 results in phosphorylation of STAT6 via the interleukin-4 receptor. Phosphorylated STAT6 proteins dimerize and translocate to the nucleus to activate transcription of target genes. In addition to inducing the expression of signature genes of alternatively activated macrophages, phosphorylated STAT6 incraeases expression of two classes of metabolic genes. First, STAT6 binds to and activates promoters of genes important in the $\beta$-oxidation of fatty acids. Second, STAT6 induces expression of transcriptional regulators (peroxisome proliferator-activated receptors $\delta$ and $\gamma$ ) and the coactivator protein PGC-1 $\beta$, which synergize with STAT6 to amplify and stabilize macrophage programs of alternative activation. Abbreviations: IL, interleukin; PGC-1 $\beta$, peroxisome proliferator-activated receptor $1 \beta$; PPAR, peroxisome proliferator-activated receptor; RXR, retinoid X receptor; STAT, signal transducer and activator of transcription; 